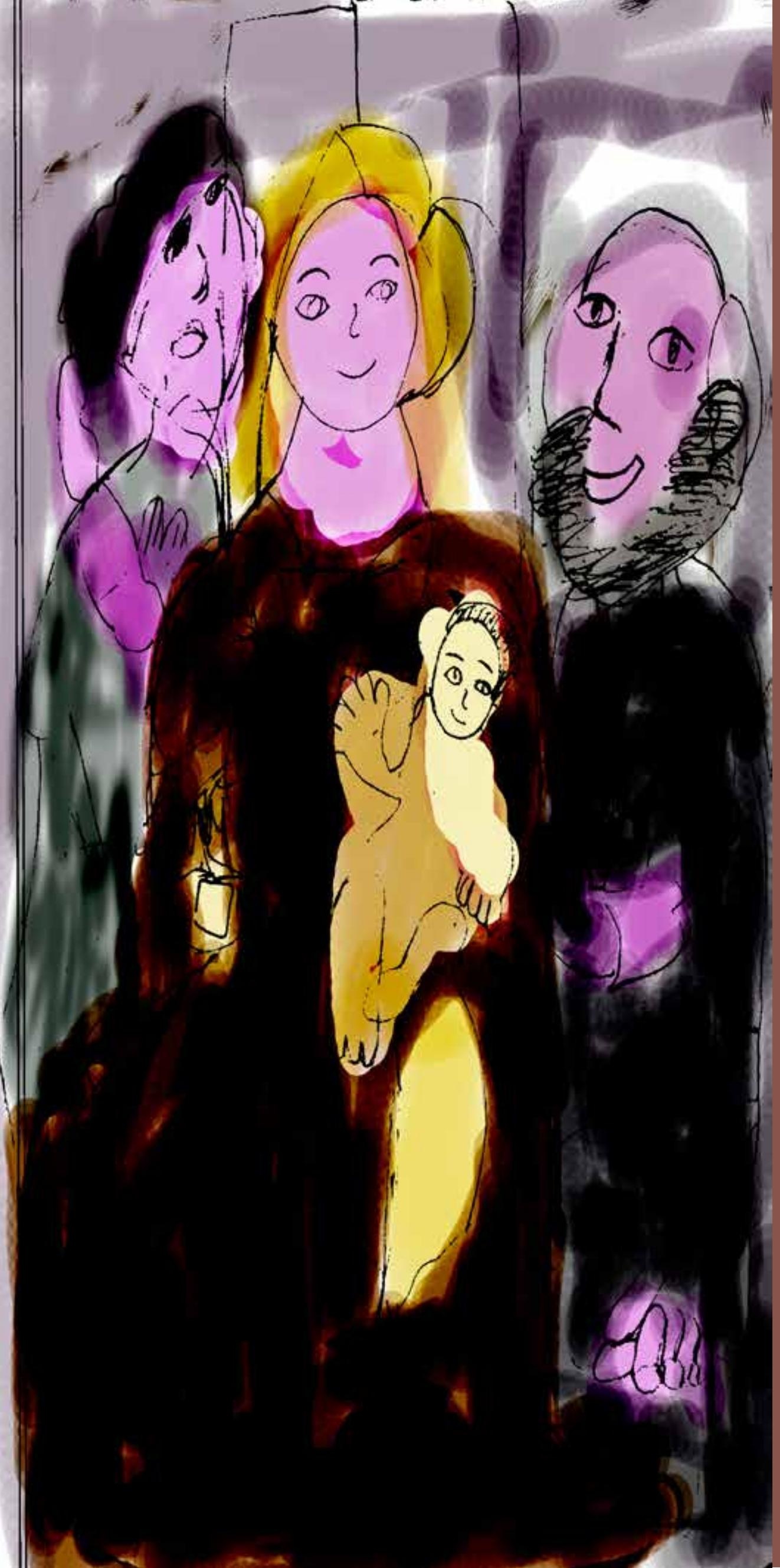

Maryluz Camargo Mendoza

Profesora Universidad Nacional, Bogotá. Colombia mcamargom@unal.edu.co

José Marín Juanías

Profesor Universidad Agustiniana.

Karla Daza

Discapacidad Intelectual

"La Virgen"

Digital 


\title{
¿ES LA LECTURA “EL ARCO IRIS” APROPIADA PARA EVALUAR DESÓRDENES DEL HABLA?
}

\section{Is the reading "el arco iris" appropriate to assess spanish speech disorders?}

\author{
Fecha de recepción: 27 de febrero de 2013 - Fecha de aprobación: 12 de mayo de 2013
}

\section{RESUMEN}

En Colombia una de las actividades más comunes a la hora de evaluar sujetos con posibles alteraciones del habla es la lectura del texto El Arco Iris. Lectura que se considera cumple con los criterios de un pasaje fonéticamente balanceado. Sin embargo, al analizar su origen, el cual corresponde a un país de lengua inglesa, surge la duda si realmente cumple con dichos criterios. En este artículo se presentan los datos resultantes tras analizar la frecuencia fonemática y la frecuencia silábica de la lectura en mención. Los resultados muestran que el texto al ser traducido del inglés, lengua en la que sí cumple criterios de balance fonético, no tiene en cuenta la distribución de los fonemas del español colombiano y presenta cargas inadecuadas en sonidos que son o muy frecuentes o poco frecuentes. Esto tiene repercusiones directas en los procesos de evaluación-diagnóstico de sujetos con alteraciones del habla, ya que una sobrecarga de sonidos poco frecuentes, puede mostrar grados de severidad de los problemas del habla mayores de los que realmente son, o por el contrario, subestimar dificultades en sonidos que por su alta frecuencia de aparición, pueden alterar de manera significativa la inteligibilidad del habla.

PALABRAS CLAVE

Desórdenes del habla, Fonética, Fonoaudiología

\section{ABSTRAC}

In Colombia, one of the most common activities when evaluating patients with possible speech alterations is the reading of the text The Rainbow. Reading which is considered to meet the criteria of a phonetically balanced passage. However, when analyzing its origin, which corresponds to an English-speaking country, the question whether it really meets those criteria arises. This paper presents the resulting data after analyzing the phonemic frequency and the syllable frequency of the intended reading. The results shows that the text, when translated from English, a language in which it does meet criteria of phonetic balance, does not take into account the phonemic distribution of the Colombian Spanish and presents inadequate loads in sounds which are either very frequent or infrequent. This fact has direct implications on assessment-diagnostic processes of subjects with impaired speech, since an overcharging of infrequent sounds, might display higher degrees of severity in speech disorders than those which really are, or conversely, underestimate difficulties in sounds which, due to their high frequency of occurrence, can significantly alter speech intelligibility.

KEYWORDS

Speech disorders, Phonetics; Speech-language Pathology 
como la secuencia de dos sonidos $/ \mathrm{k} / \mathrm{y} / \mathrm{s} /{ }^{2}$, pero que estos autores

$\mathrm{D}$ urante el proceso de evaluación - diagnóstico de los desórdenes del habla, una de las actividades que se realizan en fonoaudiología para determinar la presencia o no de un desorden, es la lectura de un texto fonéticamente balanceado'. Esto es, un texto que contiene todos los fonemas de una lengua, con sus principales variaciones sub-fonémicas, en todas las posiciones existentes dentro de la sílaba y que presenta una frecuencia relativamente proporcional a la existente en el lenguaje hablado.

En Colombia, uno de los textos más conocidos y que se considera que cumple con los criterios de un pasaje fonéticamente balanceado es El Arco Iris. Dicho pasaje se encuentra con frecuencia en formatos dedicados a consignar información propia de un proceso de evaluación-diagnóstico fonoaudiológica. De su origen se sabe que proviene de la versión original titulada como "The Rainbow" (ver Anexo para conocer las versiones en inglés y en español), la cual presenta características de un texto fonéticamente balanceado para la lengua inglesa. Su autor se desconoce, pero se tiene como fuente principal la segunda edición del libro Voice and Articulation Drillbook (Fairbanks, 1960) en la que aparece el texto en un capítulo dedicado a la evaluación de la voz. Diferentes libros y artículos proponen el uso de "The Rainbow" como una de las lecturas que deben utilizarse en la evaluación tanto de adolescentes como de adultos de habla inglesa (Smith, 2004; Gordon-Brannan, y Weiss, 2007; Shipley y McAfee, 2009; Freed, 2011).

A simple vista, El Arco Iris parece ser una traducción que pretende ser fiel a la lengua de salida, es decir al inglés, por lo que su balance fonético y fonológico para el español es algo dudoso. Con el ánimo de aportar datos que ayuden a determinar si el Arco Iris es un texto balanceado, en este artículo se presentan los resultados parciales encontrados al analizar tanto la frecuencia fonemática como la distribución de frecuencias por tipos de sílaba que presenta el texto. Pero antes de ello, se hace una breve exposición de algunos estudios acerca de los fonemas del español.

La frecuencia de los fonemas del español ha sido estudiada por diferentes autores, la mayoría de ellos en la variedad ibérica (Navarro, 1946; Alarcos, 1961; Delattre, 1965; Lloyd, 1967; Quilis y Esgueva, 1980; Llisterri y Mariño, 1993), ibérico-hispanoamericana (Rojo, 1991), argentina (Guirao y Borzone, 1972; Guirao y García, 1993) chilena (Pérez, 2003) y mexicana (Pineda, Villaseñor-Pineda, Cuétara, Castellanos \& López, 2004). El trabajo de González y Mejía (2011) es hasta el momento uno de los trabajos publicados más completos que existen acerca de la frecuencia fonemática en la variedad colombiana, describiendo 23 fonemas, 5 de ellos vocálicos y 18 consonánticos (ver Tabla 1 ). De estos últimos, dos son distintivos de la región de Colombia, el fonema fricativo palatal sonoro / $\square /$ que corresponde al fenómeno conocido como yeísmo, y el fonema africado alveopalatal sordo $/ \widehat{\mathrm{ks}} /$ que tradicionalmente se ha descrito

1. La lectura de un texto hace parte de una evaluación comportamental, que junto con una anamnesis completa, un examen físico y la realización de pruebas instrumentales, permiten al fonoaudiólogo llegar a un diagnóstico clínico.

2. Como en el caso de las palabras "éxito" o "éxtasis". los describen como uno solo.

Acerca de la frecuencia silábica son más escasos los trabajos en el español, entre los más destacados se encuentran el de Álvarez, Carreiras y De Vega (1992), Alameda y Cuetos (1995) y el de Moreno, Torre, Curto y De la Torre (2006). Los dos primeros, basados únicamente en textos escritos, y el último, tanto en corpus escritos como corpus de habla espontánea. En el trabajo de corpus orales, se encuentra que el tipo de sílaba más frecuente en español es consonante-vocal (cv) ocupando un poco más de la mitad del total de tipos de sílabas que se usan, seguido de los tipos cvc y v (ver Tabla 2).

\begin{tabular}{|c|c|c|c|}
\hline \multicolumn{4}{|c|}{ Frecuencia de los fonemas del español de Colombia } \\
\hline a & 14,57 & I & 3,42 \\
\hline e & 12,28 & u & 2,82 \\
\hline o & 11,78 & p & 2,77 \\
\hline i & 7,53 & g & 1,55 \\
\hline n & 7,29 & r & 1,04 \\
\hline r & 6,05 & X & 0,90 \\
\hline t & 5,42 & j & 0,87 \\
\hline s & 5,17 & f & 0,63 \\
\hline d & 4,13 & t & 0,46 \\
\hline b & 3,75 & N & 0,28 \\
\hline k & 3,68 & $\widehat{k s}$ & 0,05 \\
\hline m & 3,56 & & \\
\hline
\end{tabular}

Nota. Fuente: González y Mejía (2011). Frecuencia fonemática del Español de Colombia. Revista Forma y Función. Vol. 24, № 2, 69-102.

Tabla 1. Frecuencia de los fonemas del español de Colombia

Distribución de frecuencias por tipos de sílaba

\begin{tabular}{|c|c|}
\hline Tipos de sílaba & Frecuencias \\
\hline cv & 51,35 \\
\hline cvc & 18,03 \\
\hline V & 10,75 \\
\hline vc & 8,6 \\
\hline cvv & 3,37 \\
\hline cvvc & 3,31 \\
\hline ccv & 2,96 \\
\hline ccvc & 0,88 \\
\hline
\end{tabular}

Nota. Fuente: Moreno, A., Torre, D., Curto, N., y de la Torre, R. (2006). Inventario de frecuencias fonémicas y silábicas del castellano espontáneo y escrito. En: Buera, L., Lleida, E., Miguel, A. y Ortega, A. (Eds.), IV Jornadas en Tecnología del Habla. Zaragoza. Del 8 al 10 de Noviembre de 2006, 7781. Zaragoza: Universidad de Zaragoza. Consultado el 11 de enero de 2014 en http://jth2006.unizar.es/finals/4jth_116.pdf

Tabla 2. Distribución de frecuencias por tipos de sílaba 


\section{MÉTODO}

Tipo de estudio: descriptivo - cuantitativo

Objeto a estudiar: pasaje El Arco Iris en español.

- Se establecieron cada una de las frecuencias relativas tanto de los fonemas como de las sílabas haciendo uso del Software SALT -Systematic Analysis of Language Transcripts- versión 2012 (Miller e Iglesias, 2012). Para la determinación de la frecuencia fonemática se tuvieron en cuenta dos aspectos. El primero de ellos tuvo que ver con la omisión dentro de los cálculos de aquellas palabras que González y Mejía (2011) consideran que aumentan de manera significativa los cálculos de las frecuencias: que, la, y, de, el a, no, en, lo, un, le, con, porque, si, los, una, por, para, pero, aquí, muy. Y el segundo, con el análisis del fonema / $\widehat{\mathrm{ks}} /$ como un solo sonido, y no como la secuencia de dos sonidos.

- Una vez obtenidas las frecuencias arriba mencionadas, se procedió a comparar los resultados, a través del Software IBM SPSS Statistics versión 21.0, con los valores de referencia propuestos por González y Mejía (2001) y Moreno et al., (2006), tomando únicamente los datos que corresponden a los resultados encontrados en corpus orales.

\section{RESULTADOS}

\begin{tabular}{|c|c|c|c|}
\hline \multicolumn{5}{|c|}{ Frecuencia de los fonemas del Arco Iris } \\
\hline a & 13,87 & d & 2,91 \\
\hline o & 11,30 & b & 1,88 \\
\hline S & 10,96 & $\mathrm{~g}$ & 1,88 \\
\hline e & 10,45 & j & 1,37 \\
\hline i & 7,71 & $\mathrm{p}$ & 1,20 \\
\hline r & 6,85 & $\mathrm{f}$ & 1,20 \\
\hline n & 5,99 & $\mathrm{ks}$ & 0,86 \\
\hline I & 4,62 & $\mathrm{r}$ & 0,51 \\
\hline t & 4,11 & $\mathrm{x}$ & 0,34 \\
\hline m & 4,11 & $\mathrm{t}$ & 0,34 \\
\hline k & 3,94 & $\mathrm{n}$ & 0,17 \\
\hline u & 3,42 & & \\
\hline
\end{tabular}

Tabla 3. Frecuencia de los fonemas del Arco Iris.

Las Tablas 3 y 4 así como las Figuras 1 y 2 presentan los datos resultantes:

\section{DISCUSIÓN}

Como se puede observar, los valores de las Tablas 1 y 2 difieren de manera considerable de los valores de las Tablas 3 y 4 , que son los valores de referencia que se tomaron en los análisis. Se observa de manera clara, una sobrecarga principalmente del fonema /s/, así como también de /r/, /l/, /f/, /m/ y / $\widehat{\mathrm{ks}} /$, y de forma contraria, una reducción de la frecuencia de los fonemas vocálicos /a/, /o/ y /e/, así como de los consonánticos $/ \mathrm{n} /, / \mathrm{t} /, / \mathrm{d} /, / \mathrm{b} /, / \mathrm{p} / \mathrm{y} / \mathrm{x} /$. El tipo de sílaba cv que según Moreno et al., (2006) en el español es la más abundante, presenta una disminución en su frecuencia. Mientras que otros tipos de sílaba, no tan frecuentes, cvc, vc, ccv, v y vc presentan una frecuencia aumentada o sobrecargada.

\begin{tabular}{|c|c|}
\hline \multicolumn{2}{|c|}{ Distribución de frecuencias por tipos de sílaba en El Arco Iris } \\
\hline Tipos de sílaba & Frecuencias \\
\hline cv & 39,8 \\
\hline cvc & 22,6 \\
\hline v & 11,7 \\
\hline vc & 11,7 \\
\hline cvv & 3,16 \\
\hline cvvc & 2,75 \\
\hline ccv & 4,36 \\
\hline ccvc & 2,75 \\
\hline vv & 0,78 \\
\hline ccvv & 0,4 \\
\hline
\end{tabular}

Tabla 4. Distribución de frecuencias por tipos de sílaba en El Arco Iris.

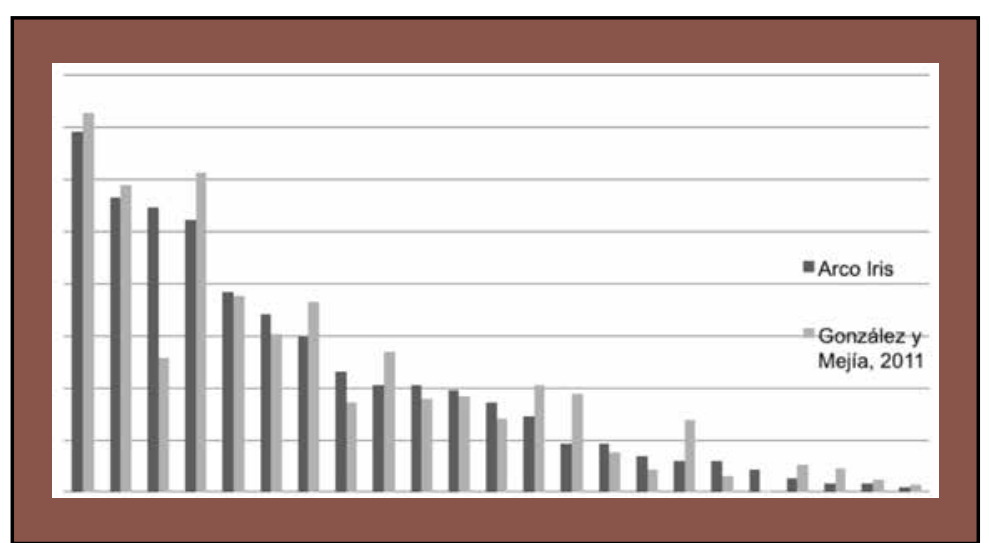

Figura 1. Frecuencia fonemática del español colombiano vs El Arco Iris

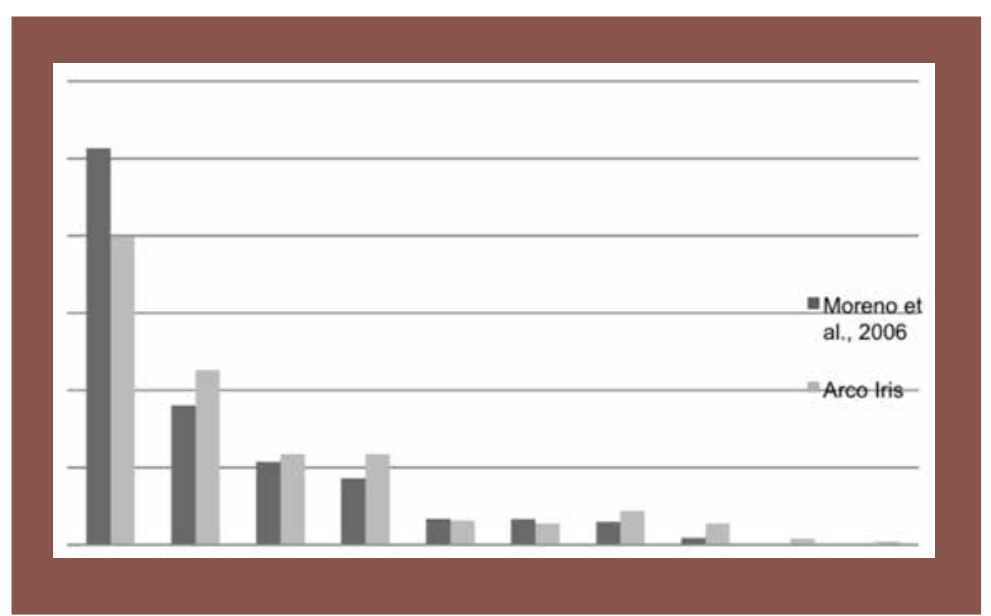

Figura 2. Distribución de frecuencias por tipos de sílaba del español vs El Arco Iris 
El Arco Iris no fue elaborado para el español, lo fue para el inglés, por lo que cualquier uso que se haga del texto debe ser diferente al de apoyar un proceso de evaluación-diagnóstico de los desórdenes del habla. Textos como este son usualmente utilizados en las prácticas clínicas, lo cual es un desacierto en la medida que pueden mostrar grados de severidad de los problemas del habla mayores de los que realmente son, o por el contrario, subestimar dificultades en sonidos que por su alta frecuencia de aparición pueden alterar de manera significativa la inteligibilidad del habla. Así por ejemplo, una persona con dificultades en la producción del fonema $/ \widehat{\mathrm{ks}} /$, con El Arco Iris va a mostrar que presenta un problema importante al no producir correctamente las palabras meta que allí se presentan. Sin embargo, los datos de González y Mejía (2011) muestran cómo la frecuencia de dicho fonema en el español colombiano es mínima. Por el contrario, a una persona con dificultades en los fonemas oclusivos $/ \mathrm{t} /, / \mathrm{d} /, / \mathrm{b} /, / \mathrm{p} /$, se le puede llegar a minimizar su problema al no encontrar tan notoria dicha dificultad, debido a la baja frecuencia con la que aparecen en El Arco Iris.

Por fortuna, textos como el que se ha analizado, son una pequeña parte de la evaluación comportamental que se realiza en fonoaudiología, pero no por ello se debe ignorar la importancia de contar con materiales adecuados, confiables, basados en las características propias de las culturas y las lenguas.

\section{CONCLUSIÓN}

En este documento se presentaron los resultados encontrados al analizar el pasaje El Arco Iris, como una manera de aproximarse a su realidad fonética y silábica, para así reflexionar acerca de la pertinencia de usarlo o no en las actividades de evaluación- diagnóstico de los desórdenes del habla. Aun cuando este estudio no se detuvo a examinar si presentaba las variaciones sub-fonémicas, ni se revisaron las posiciones existentes de cada uno de los fonemas dentro de la sílaba, se puede decir que el texto no representa la realidad fonética ni fonológica del español en general, ni del español colombiano, en particular. Por lo que es conveniente que académicos e investigadores interesados en las patologías del habla, diseñen textos apropiados para la población colombiana y los pongan a disposición de la fonoaudiología. Esto evitaría el uso de textos como El Arco Iris, que pueden llevar a que los profesionales cometan errores en los procesos de evaluación - diagnóstico. Mientras esto sucede, es preferible usar textos que, aunque no tengan un balance fonético para el español colombiano, si lo presenten para otra variedad del español ${ }^{3}$.
VERSIÓN EN INGLÉS

When the sunlight strikes raindrops in the air, they act as a prism and form a rainbow. The rainbow is a division of white light into many beautiful colors These take the shape of a long round arch, with its path high above, and its two ends apparently beyond the horizon.

There is, according to legend, a boiling pot of gold at one end. People look but no one ever finds it. When a man looks for something beyond his reach, his friends say he is looking for the pot of gold at the end of the rainbow.

Throughout the centuries people have explained the rainbow in various ways. Some have accepted it as a miracle without physical explanation. To the Hebrews it was a token that there would be no more universal floods. The Greeks used to imagine that it was a sign from the gods to foretell war or heavy rain. The Norsemen considered the rainbow as a bridge over which the gods passed from earth to their home in the sky. Others have tried to explain the phenomenon physically. Aristotle thought that the rainbow was caused by reflection of the sun's rays by the rain.
VERSIÓN EN ESPAÑOL

Cuando la luz del sol atraviesa las gotas de lluvia en el aire, ésta actúa como un prisma y forma un arco iris. El arco iris es una división de la luz blanca en muchos colores hermosos. Estos toman la forma de un arco alto con dos extremos aparentemente más allá del horizonte.

Hay, de acuerdo a la leyenda, una olla de oro hirviente en uno de los extremos. La gente busca, pero nadie la encuentra. Cuando un hombre busca algo más allá de su alcance, sus amigos dicen que está buscando la olla de oro al final del arco iris.

A través de los siglos los hombres han explicado el arco iris de muchas formas. Algunos lo han aceptado como un milagro. Para los hebreos era una señal de que no habría más inundaciones universales. Los griegos imaginaban que era un signo de los dioses desde la tierra hasta el cielo. Otros hombres han explicado el fenómeno físicamente. Aristóteles pensó que el arco iris se debía a la reflexión de los rayos del sol en la lluvia.

3. Joaquím Llisterri en su página de internet http://liceu.uab.es/ joaquim/phonetics/fon_esp/Textos_equilibrio_fonetico_espanol.html, presenta dos textos en español balanceados fonéticamente que pueden ser de utilidad. 


\section{Referencia:}

\section{Las referencias a otras obras son una parte muy importante en la literatura científica; ya que estas permiten conocer más sobre los autores y mantener vivas sus voces dentro del texto.}

Alameda, J., \& Cuetos, F. (1995) Diccionario de frecuencias de las unidades lingüísticas del castellano. Oviedo: Servicio de publicaciones de la Universidad de Oviedo.

\section{Alarcos, E. (1961). Fonología Española. Madrid: Gredos.}

Álvarez, C., Carreiras, M. \& de Vega, M. (1992). Estudio estadístico de la ortografía castellana: (1) la frecuencia silábica. Cognitiva 4, 75-105.

Delatre, P. (1965). Comparing the phonetic features of English, German, Spanish and French. Heidelberg: J.Groos.

Fairbanks, G. (1960). Voice and Articulation Drillbook. Baltimore: Williams \& Wilkins.

Freed, D. (2011). Motor Speech Disorders: Diagnosis \& Treatment (2nd Edition) New York: Cengage Learning.

González \& Mejía (2011). Frecuencia fonemática del Español de Colombia. Revista Forma y Función. Vol. 24, № 2, 69-102.

Gordon-Brannan, M. \& Weiss, C. (2007). Clinic management of articulatory and phonologic disorders (3rd ed.), Baltimore: Lippincott Williams \& Wilkins.

Guirao, M. \& Borzone de Manrique, A. (1972). Fonemas, sílabas y palabras del español de Buenos Aires. Filología, XVI, 135-165.

Guirao, M. \& García, M. (1993). Estudio estadístico del español. Buenos Aires: Consejo Nacional de Investigaciones Científicas y Técnicas.

Llisterri, J. \& Mariño, J. (1993). Spanish adaptation of SAMPA and automatic phonetic transcription. SAM-A/UPC/001/v1. Esprit project 6819. (SAM-A Speech Technology Assessment in Multilingual Applications). Consultado el 18 de enero de 2014 en http://liceu.uab.cat/ -joaquim/publicacions/ SAMPA_Spanish_93.pdf

Loyd, P. (1967). A statistical study of the structure of Spanish syllable. Linguistics, Vol. 37, 58-72.
Miller, J. \& Iglesias, A. (2012). Systematic Analysis of Language Transcripts (SALT), Version 2012 [Computer Software], SALT Software, LLC.

Moreno, A., Torre, D., Curto, N. \& de la Torre, R. (2006). Inventario de frecuencias fonémicas y silábicas del castellano espontáneo y escrito. En: Buera, L., Lleida, E., Miguel, A. y Ortega, A. (Eds.), IV Jornadas en Tecnología del Habla. Zaragoza. Del 8 al 10 de Noviembre de 2006, 77-81. Zaragoza: Universidad de Zaragoza. Consultado el 11 de enero de 2014 en http:// jth2006.unizar.es/finals/4jth_116.pdf

Navarro, T. (1946). Estudios de Fonología Española. Syracruse. New York: University Press.

Pérez, H. (2003). Frecuencia de fonemas. e-rthabla, 1. Consultado el 10 de enero de 2014 en http://gth-www.die.upm.es/numeros/N1/N1_A4.pdf

Pineda, L., Villaseñor-Pineda, L., Cuétara, J., Castellanos, H., \& López, I. (2004). DIMEx100: A New Phonetic and Speech Corpus for Mexican Spanish. En Lemaître, C., Reyes, A. y González, J. (Eds.), Iberamia, 2004, LNAI 3315, 974-983. Consultado el 20 de enero de 2014 en http://pdf.aminer.org/o0o/262/029/dimex_a_new_phonetic_and_speech_corpus_for_ mexican_spanish.pdf

Rojo, G. (1991) Frecuencia de fonemas en el español actual en Brea, M. y Fernández, F. (Coords.). Homenaxe ó profesor Constantino García. Santiago de Compostela: Universidade de Santiago. p.451-457.

Shipley, H. \& McAfee, J. (2009). Assessment in Speech-Language Pathology: A Resource Manual (4th Edition). New York: Delmar Cengage Learning.

Smit, A. (2004). Articulation and Phonology. Resource Guide for School-Age Children and Adults. New York: Thomson Delmar Learning.

Quilis, A. \& Esgueva, M. (1980). Frecuencia de fonemas en el español hablado. Lingüística Española Actual, Vol. 2, p. 1-25. 